\title{
Merging virtual world with data sciences
}

\begin{abstract}
Virtual world is generating large amount of data. Gaming industry is one of the actors in the production of huge amount, variety and velocity of data. Merging of virtual world with data sciences can give better understanding of the underlying mechanics for all the stakeholders. To do so the idea is to bring forth the rich data model known as Resource Description Framework (RDF) of Semantic Web to become a middle source of bridging Big-data and Virtual platforms.
\end{abstract}

Keywords: data science, big data, virtual reality, artificial intelligence
Volume 2 Issue 2 - 2017

\author{
Kaleem Razzaq Malik, ${ }^{1,2}$ Muhammad Farhan ${ }^{1,2}$ \\ 'Department of Computer Science and Engineering, University \\ of Engineering and Technology, Pakistan \\ ${ }^{2}$ Department of Computer Science, COMSATS Institute of \\ Information Technology, Pakistan
}

\begin{abstract}
Correspondence: Kaleem Razzaq Malik, Department of Computer Science and Engineering, University of Engineering and Technology, Pakistan, Email krmalik@gmail.com
\end{abstract}

Received: March 25, 2017 | Published: March 30, 2017
Abbreviations: AI, artificial intelligence; VR, virtual reality; $\mathrm{AR}$, augmented reality; IOT, internet of things

\section{Introduction}

Data has become humongous even when coming from single source like mobile consisting of many forms of sensors included. Data coming from all different and similar sources distributed over the globe makes magnitude harder to process up-to a needed scale. Big data and Deep learning had become standard in providing well-known solutions built-up using algorithms and techniques like association rule, classification tree, genetic algorithms, machine learning, regression and sentimental analysis etc. in resolving matching issues for learning the outcome effects. ${ }^{1}$ Now with the involvement of sensors and automation in generating data complicates everything. Predicting results to overcome current era of ever enhancing demands and getting real-time visualization brings the need of feature like hierarchal modeling of data to be improved such that to bring down the complexity of data capturing and analyzing process. Virtualization brings opportunity of predicting happening with the help of the chances of lossless alternative and optimal option selection. The same is true for the eLearning opportunities. ${ }^{2}$

Semantic web comes with the solution of relativity built-in the data model to support hierarchal linking of data resources. ${ }^{3}$ Due to the part of its capability as unique identification for each data resource brings up classification to produce instances of data as forming a triple of Source, Predicate and Object. Where predicate shows the relationship between the two source and object data. Few of its examples can be seen as source being a variable where object becomes the value. Or source as a class and object becomes the instance. This can go on in representing data relativity with each other forming linking among within or with other data forms. This is also called linked data. Similar representation is used when defining the rules for Artificial Intelligence (AI) to depict a result. That is why semantic web based representation is compatible and useful resource when making intelligence part of the web. ${ }^{3,4}$ It brings motif of thought which still lakes to its full extent till now. This makes it a research area to work in but the trend is have moved from data form to data capacity coverage which brought big data by combining the concepts of computing and semantic web data representation together as to distribute data as clustering unit to be processed among all available resources. The use of AI and related technologies for the analysis of eLearning content can result in data analytics, which is a merger of virtual world and data science. ${ }^{5}$ Now let's look at current required environment getting adopted by world for learning on problem's resolution is by using and trying to bring forth the utilization of technologies like Virtual Reality (VR) and Augmented Reality (AR). Whereas, if concept of big data, IOT and machine learning combined with semantic web data representation brought in VR and AR environment for analyzing and resolving issues can bring dramatic change in the field of science overall (Figure 1).

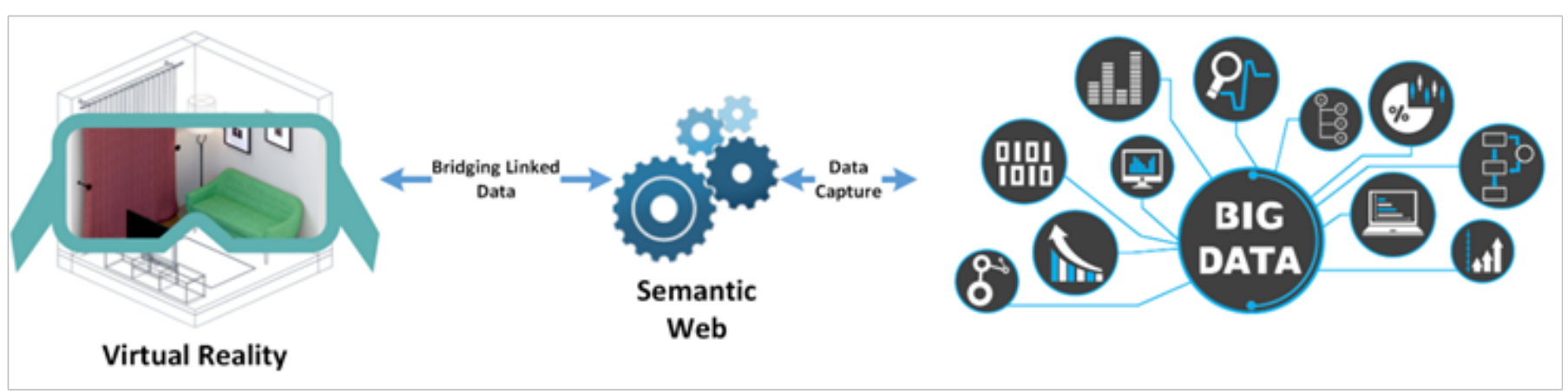

Figure I Semantic Web as merging source of the VR with Big data.

Specially, it may be more effective in the fields of learning, eLearning, math, physics, chemistry, and biological study. For instance, surgical operates can become similar to fiction like 3D operations where machine can be deployed to support the task remotely if the doctor is not available on spot physically. On the bases of prediction and history mapped in machine learned system can be used to perform highly adoptive to the situation by linking the instances using triple form of data. Another example can be teaching of different topics 
where multi-agent based system can support in predicting suitable teaching material and technique in support of teachers in the field of education either conventional or eLearning. In both examples VR or AR can be proved highly useful as being bringing the environment remotely to understand the situation in learning phase of the brain. The video content used in eLearning setup is a sort of virtual world due to the absence of teacher or moderator so the assessment using data collection methods. This scenario also refers to the merger and both the domains. ${ }^{6}$

\section{Conclusion}

In big data, data originates from many distributed and different sources in the shape of audio, video, text and sound on the bases of real time; which makes it massive and complex for traditional systems to handle. For this, data representation is required in the form of semantically-enriched for better utilization but keeping it simplified is essential. Late research demonstrates that fast data assets in the wild are developing at an amazing rate. The fast build number of data assets has conveyed a critical need to create insightful techniques to compose and procedure them. In this paper, the Semantic Representation model is utilized for arranging approaching data assets. Numerical demonstrating is intended to set up related relations among different assets going for expanding the approximately associated system of no semantics to an affiliation rich system.

\section{Acknowledgements}

None.

\section{Conflict of interest}

The author declares no conflict of interest.

\section{References}

1. Malik KR, Ahmad T, Farhan M, et al. Big-data: transformation from heterogeneous data to semantically-enriched simplified data. Multimedia Tools and Applications. 2016;75(20):12727-12747.

2. Farhan M, Aslam M, Jabbar S, et al. Multimedia based qualitative assessment methodology in eLearning: student teacher engagement analysis. Multimedia Tools and Applications. 2016. p. 1-15.

3. Malik KR, Ahmad T. Technique for Transformation of data from RDB to XML Then to RDF. Web semantics for textual and visual information retrieval. IGI Global. 2017. p. 70-91.

4. Malik KR, Ahmad T, Farhan M, et al. Multiagent semantical annotation enhancement model for IoT-based energy-aware data. International journal of distributed sensor networks; 2016.

5. Muhammad F, Muhammad A. An interactive assessment framework for visual engagement: statistical analysis of a TEDx video. EURASIA Journal of Mathematics, Science and Technology Education. 2017;13:11071119.

6. Farhan M, Aslam M, Jabbar S, et al. Real-time imaging-based assessment model for improving teaching performance and student experience in e-learning. Journal of Real-Time Image Processing. 2017. p. 1-14. 